\title{
Colour-singlet Strangelets at Finite Temperature
}

\author{
M. G. Mustafa * and A. Ansari * \\ Institute of Physics, Bhubaneswar-751005, India.
}

(October 23, 2018)

\begin{abstract}
Considering massless $u$ and $d$ quarks, and massive $(150 \mathrm{MeV}) s$ quarks in a bag with the bag pressure constant $B^{1 / 4}=145 \mathrm{MeV}$, a colour-singlet grand canonical partition function is constructed for temperatures $T=1-30 \mathrm{MeV}$. Then the stability of finite size strangelets is studied minimizing the free energy as a function of the radius of the bag. The colour-singlet restriction has several profound effects when compared to colour unprojected case: (1) Now bulk energy per baryon is increased by about $250 \mathrm{MeV}$ making the strange quark matter unbound. (2) The shell structures are more pronounced (deeper). (3) Positions of the shell closure are shifted to lower $A$-values, the first deepest one occuring at $A=2$, famous $H$-particle ! (4) The shell structure at $A=2$ vanishes only at $T \sim 30 \mathrm{MeV}$, though for higher $A$-values it happens so at $T \sim 20 \mathrm{MeV}$.
\end{abstract}

PACS numbers: 12.38.Aw,12.38.Mh, 12.40.Aa, 24.85.+p 
Witten conjectured in 1984 [1] that the strange quark matter (consisting of roughly equal number of $u, d$ and $s$ quarks) might be absolutely stable compared to normal matter in which iorn is the most stable. The possibility of stable/metastable droplets (strangelets) was also suggested. A few years later Greiner and coworkers [2,3] also predicted that during the phase transition from quark-gluon plasma (formed in relativistic heavy ion collisions) to hadrons metastable droplets could be formed with baryon number $A \sim 10-30$. Experimental search for strangelets is still going on in relativistic heavy ion collisions [4]. Recently Madsen [5 6] has written several papers on the stability of strangelets following liquid drop model as well as shell model. Gilson and Jaffe [8] have studied the stability of small strangelets, with $A<100$, considering independent particle shell model approach with massless $u$ and $d$ quarks, and massive $s$ quark $\left(m_{s}=150 \mathrm{MeV}\right)$ confined in a bag. At zero temperature clear shell structures are found at certain baryon numbers emplying the possibility of metastable strangelets.

In a very recent paper [9] we have extended the work of Gilson and Jaffe [8] to finite temperature. Using the eigen modes of $u, d$ and $s$ quarks in the MIT bag with the bag constant $B^{1 / 4}=145 \mathrm{MeV}$ we have studied in Ref. [9] the thermodynamic properties of small strangelets at finite temperature $T=0.5-30 \mathrm{MeV}$. This scheme of calculation has the advantage that at $T=0.5 \mathrm{MeV}$ the shell structure of strangelets is as transparent as those of Ref. [8] at zero temperarure. At the same time the study can be made at higher temperatures. There we found that the shell structure melts away at $T \geq 10 \mathrm{MeV}$. In this study of ours and as well as of others, the strangelets were not constrained to be colour-singlet which should be natural for a bound (confined) system. In those studies the statistical description of many-body quantum systems are based on unrestricted grand canonical ensemble average. One is usually forced to employ approximate one-body statistical operators with broken symmetries. Then in order to restore the symmetry one needs a projected statistics with good quantum numbers, like angular momentum, particle numbers, parity, colour etc. In the present study we want to have the correct colour symmetry, that is, we construct a colour-singlet partition function to study the stability of finite size strangelets at finite 
temperature. For a single baryon we have already made such studies in the past [10]. This is done following the standard projection technique in the $S U(3)$ colour gauge space [11.

The grand canonical partition function for a system of quarks, anti-quarks and gluons is given by

$$
\mathcal{Z}_{j}(\beta, V)=\operatorname{Tr}\left(\hat{\mathcal{P}}_{j} e^{-\beta \hat{H}}\right)
$$

where $\beta=1 / T, \hat{H}$ is the Hamiltonian of the system and $\mathcal{P}_{j}$ is a projection operator for a symmetry group $\mathcal{G}$ (compact Lie group) having a unitary representation $\hat{U}(g)$ in a Hilbert space $\mathcal{H}$,

$$
\hat{\mathcal{P}}_{j}=d_{j} \int_{\mathcal{G}} d \mu(g) \chi_{j}^{\star}(g) \hat{U}(g)
$$

where $d \mu(g)$ is the normalized Haar measure in $\mathcal{G} . d_{j}$ and $\chi_{j}$, are, respectively, the dimension and the character of the irreducible representation $j$ of $\mathcal{G}$. For $S U(3)$ colour-singlet configuration $d_{j}=1$ and $\chi_{j}=1$, and the partition function for quark-gluon system becomes

$$
\mathcal{Z}_{C}(\beta, V)=\int_{S U(3)} d \mu(g) \operatorname{Tr}\left(\hat{U}(g) e^{-\beta \hat{H}}\right)
$$

where

$$
\int_{S U\left(N_{C}\right)} d \mu(g)=\frac{1}{N_{C} !}\left(\prod_{i=1}^{N_{C}-1} \int_{-\pi}^{\pi} \frac{d \theta_{i}}{2 \pi}\right)\left[\prod_{j<k}^{N_{C}}\left(2 \sin \frac{\theta_{j}-\theta_{k}}{2}\right)^{2}\right]
$$

with $N_{C}=3$ for $S U(3)$ colour group. The chemical potential dependent partition function can be written (more details can be found in Refs. [10,11]) as

$$
\mathcal{Z}_{C}\left(\beta, V, \mu_{q}\right)=\int_{S U(3)} d \mu(g) e^{\Theta}
$$

with

$$
\begin{aligned}
\Theta= & \sum_{\alpha}\left[\ln \left\{1+\chi_{q}(g) e^{-\beta\left(\epsilon_{q}^{\alpha}-\mu_{q}\right)}\right\}+\ln \left\{1+\chi_{\bar{q}}^{\star}(g) e^{-\beta\left(\epsilon_{\bar{q}}^{\alpha}+\mu_{q}\right)}\right\}\right. \\
& \left.-\ln \left\{1-\chi_{\text {adj }}(g) e^{-\beta \epsilon_{g}^{\alpha}}\right\}\right]
\end{aligned}
$$




$$
\begin{gathered}
\text { where } \chi_{q}(g)=\sum_{i=1}^{3} e^{i \theta_{i}}, \\
\chi_{\text {adj }}(g)=\left|\chi_{q}(g)\right|^{2}-1=2+\sum_{i<j}^{3} \cos \left(\theta_{i}-\theta_{j}\right),
\end{gathered}
$$

are, respectively, the characters of the group in the fundamental (quarks) and adjoint (gluons) representations. $\theta_{i}$ is a class parameter obeying the constraint $\sum_{i=1}^{3} \theta_{i}=0(\bmod 2 \pi)$. $\alpha$ runs over discrete single-particle states of quarks and gluons confined in a bag with $\epsilon_{q}^{\alpha}$ representing quark energies and $\epsilon_{g}^{\alpha}$ the gluon energies. The upper limit of the summation $\alpha$ depends upon the temperature considered.

The colour unprojected partition function is obtained by putting $\hat{\mathcal{P}}_{j}=1$ in eq.(11)

$$
\mathcal{Z}_{U}(\beta, V)=\operatorname{Tr}\left(e^{-\beta \hat{H}}\right)
$$

Then for a system of quarks, anti-quarks and gluons

$$
\mathcal{Z}_{U}\left(\beta, V, \mu_{q}\right)=e^{\Theta}
$$

where

$$
\Theta=\sum_{\alpha}\left[\ln \left\{1+e^{-\left(\beta \epsilon_{q}^{\alpha}-\mu_{q}\right)}\right\}+\ln \left\{1+e^{-\left(\beta \epsilon_{\bar{q}}^{\alpha}+\mu_{q}\right)}\right\}-\ln \left\{1-e^{-\beta \epsilon_{g}^{\alpha}}\right\}\right]
$$

It is to be noted that in eq.(8) trace is performed with equal weight without recourse to the corresponding colour group having unitary representation.

Unlike in Ref.[9] we do not require strangelets to be charge neutral. However we ignore the Coulomb corrections because charge $(Z)$ is very small for small $A, Z<<A$. We also exclude here, like in Ref. [9], the phenomenological zero point energy term due to great uncertainty in the choice of the value of the parameter involved. The baryon number $A$ is fixed by adjusting the quark chemical potential such that the excess number of $q$ over $\bar{q}$ is $3 A$, viz.

$$
\triangle N_{q}=N_{q}-N_{\bar{q}}=\left(N_{u}-N_{\bar{u}}\right)+\left(N_{d}-N_{\bar{d}}\right)+\left(N_{s}-N_{\bar{s}}\right)=3 A
$$

where 


$$
N_{i}=T \frac{\partial}{\partial \mu_{i}}\left(\ln \mathcal{Z}_{\left(\begin{array}{c}
U \\
C
\end{array}\right)}\right)
$$

with $i=q, \bar{q}$ and $\mu_{\bar{q}}=-\mu_{q}$.

The energy, $E$ and free energy, $F$ of the system can be written as

$$
\begin{gathered}
E(T, R)=T^{2} \frac{\partial}{\partial T}\left(\ln \mathcal{Z}_{\left(\begin{array}{l}
U \\
C
\end{array}\right)}\right)+\mu_{q} \triangle N_{q}+B V, \\
F(T, R)=-T \ln \mathcal{Z}_{\left(\begin{array}{l}
U \\
C
\end{array}\right)}+\mu_{q} \triangle N_{q}+B V
\end{gathered}
$$

where $B V$ is the bag volume energy [12].

The pressure generated by the participants gas

$$
P=-\left(\frac{\partial}{\partial V} F(T, R)\right)_{T, \triangle N_{q}},
$$

is balanced by the bag pressure constant, $B$ leading to the stability condition of the system. Then finally the equilibrium energy of the system is given by

$$
E\left(T, R_{0}\right)=4 B V=\frac{16}{3} \pi B R_{0}^{3}
$$

Actually $R_{0}$ represents the equilibrium value of the radius at which the free energy (14) is minimum.

First of all we may remark that at temperatures considered here the contributions from anti-quarks and gluons are insignificant. Now we can discuss our results. In Fig. 1a we show the variation of the baryon energy per particle $(E / A)$ as a function of $A$ for colour unprojected case at various temperatures as indicated there. In Fig. 1b same is shown for colour projected (colour-singlet) case. The lowest temperature considered is $T=1 \mathrm{MeV}$ as we are mainly interested in comparative studies. Looking at Figs. 1a and 1b the following features constitute our main results:

1. For the colour-singlet case the bulk energy per baryon has increased to about $1.1 \mathrm{GeV}$ so that strange quark matter becomes unbound in bulk for $B^{1 / 4}=145 \mathrm{MeV}$ (for colour unprojected case it is $850 \mathrm{MeV}$ ). The metastable strangelets could eventually decay into nuclei. We are not discussing here the various kinds of decay modes as those are discussed in Ref. [8] by Gilson and Jaffe. 
2. The first shell structure appears at $A=2$ instead of at 6 when colour-singlet restriction is not imposed. This is a very significant change as now at $A=2$ the number of $u, d$ and $s$ quarks each is 2 . Otherwise there are three $u$-quarks, three $d$-quarks and zero $s$ quarks (see Fig. 2). This clearly corresponds to metastable $H$ particle with mass $\approx 2.5 \mathrm{GeV}$.

3. For higher baryon numbers also the shell positions have shifted to lower $A$-values with more stable shell effects. These are $A=8,14,18,28 \ldots$ The positions of shells are likely to be very important in the range $A \leq 30$ as experimental search to detect stable/metastable strangelets are going on in relativistic heavy ion collisions [4].

4. In Fig. $1 \mathrm{~b}$ shell structures for higher $A$-values vanish at $T \geq 20 \mathrm{MeV}$ compared to about $10 \mathrm{MeV}$ in Fig. 1a. Infact for colour-singlet case at $A=2$ the shell structure disappears only at $T \sim 30 \mathrm{MeV}$ whereas that for unprojected case at $A=6$ it vanishes only at $T \sim 20 \mathrm{MeV}$.

In Figs. 2a and $2 \mathrm{~b}$ are displayed the number of $u, d$ and $s$ quarks per baryon as a function of $A$ for colour unprojected and projected cases, respectively. For the colour-singlet strangelets the fraction of $s$ quarks is relatively high for very small sized strangelets, $A<15$, there being a strong similiarity at $A=2$ in the colour projected case and $A=6$ in the colour unprojected case. In Figs. 3a and 3b we show the variation of charge per baryon. In these figures shell closures are very clearly seen with net charge being zero there. This also implies that for these metastable strangelets $(E / Z)$ ratio is very large, as usually expected [13, 14. The strangelets with closed shells are relatively more stable and chemically less reactive.

To conclude we have shown that colour-singlet restriction on strangelets has a profound effect. The prediction of metastable $H$ particle comes out as a natural consequence of this. Next we plan to study the effect of quadruploe shape deformation on the stability of colour-singlet strangelets. 


\section{REFERENCES}

* $\quad$ e-mail: mustafa / ansari@iopb.ernet.in

[1] E. Witten, Phys. Rev. D30, 272 (1984)

[2] C. Greiner, D. H. Rischke, H. Stocker, and P. Koch, Phys. Rev. D38, 2797 (1988)

[3] C. Greiner, P. Koch, and H. Stocker, Phys. Rev. Lett. 58, 1825 (1987); Phys. Rev. D44 $3517(1991)$

[4] A. Rusek et al., Nucl. Phys. A585, 59 (1995)

[5] M. L. Olesen, and J. Madsen, Phys. Rev. D47, 2313 (1993)

[6] J. Madsen, Phys. Rev. Lett. 70, 391 (1993)

[7] J. Madsen, Phys. Rev. D 50, 3328 (1994)

[8] E. P. Gilson, and R. L. Jaffe, Phys. Rev. Lett. 71, 332 (1993)

[9] M. G. Mustafa, and A. Ansari, Preprint No. IP/BBSR/95-46; nucl-th/9505044

[10] A. Ansari, and M. G. Mustafa, Nucl. Phys. A 539, 751 (1992); M. G. Mustafa, Phys. Lett. B318, 517 (1994)

[11] G. Auberson et al., J. Math. Phys. 27, 1658 (1986)

[12] T. A. DeGrand, R. L. Jaffe, K. Johnson, and J. Kiskis, Phys. Rev. D12, 2060 (1975)

[13] G. B. Frankline, Nucl. Phys. A585, 83c (1995)

[14] J. Belz et al., Nucl. Phys. A585, 97 $(1995)$ 


\section{FIGURES}

FIG. 1. Energy per baryon $(E / A)$ as a function of baryon number $A$ for different temperatures $(T=1,10,15,20 \mathrm{MeV})$ with $B^{1 / 4}=145 \mathrm{MeV}$ and $s$-quark mass $m_{s}=150 \mathrm{MeV}$ :(a) Colour unprojected case, (b) Colour projected(colour-singlet) case.

FIG. 2. Quark-fraction as a function of $A$ for different temperatures $(T=1,15,30 \mathrm{MeV})$

with $B^{1 / 4}=145 \mathrm{MeV}$ and $s$-quark mass $m_{s}=150 \mathrm{MeV}$ (a) Colour unprojected case, (b) Colour projected case.

FIG. 3. Same as Fig. 2 for charge-fraction 
This figure "fig1-1.png" is available in "png" format from: http://arxiv.org/ps/nucl-th/9506021v1 
This figure "fig1-2.png" is available in "png" format from: http://arxiv.org/ps/nucl-th/9506021v1 
This figure "fig1-3.png" is available in "png" format from: http://arxiv.org/ps/nucl-th/9506021v1 
This figure "fig1-4.png" is available in "png" format from: http://arxiv.org/ps/nucl-th/9506021v1 
This figure "fig1-5.png" is available in "png" format from: http://arxiv.org/ps/nucl-th/9506021v1 
This figure "fig1-6.png" is available in "png" format from: http://arxiv.org/ps/nucl-th/9506021v1 